respectively), reduction in glomerular filtration rate $\left(5.8 \mathrm{ml} / \mathrm{min} / 1.73 \mathrm{~m}^{2} \mathrm{vs} 11.7 \mathrm{ml} / \mathrm{min} / 1.73 \mathrm{~m}^{2}\right.$, respectively) or median change in height. Three patients showed signs of mild CNI nephrotoxicity, and tacrolimus was discontinued in four children because of lack of response, glucose intolerance or hypertension. Antihypertensives were required for 11 of 31 ciclosporin treatment-years and for 22 of 40 tacrolimus treatment-years. The authors conclude that switching from ciclosporin to tacrolimus does not improve the management of severe SDNS in children.

Original article Sinha MD et al. (2006) Treatment of severe steroid-dependent nephrotic syndrome (SDNS) in children with tacrolimus. Nephrol Dial Transplant 21: 1848-1854

\section{Meta-analysis of ACE inhibitor plus ARB combination therapy for proteinuric renal disease}

Inhibition of the renin-angiotensin system using either an angiotensin-converting-enzyme (ACE) inhibitor or an angiotensin-receptor blocker (ARB) effectively decreases rates of urinary protein excretion and slows progression of proteinuric renal disease. Some studies have indicated that concomitant administration of an ACE inhibitor and an ARB results in more complete blockade of the reninangiotensin system than either compound alone, but the safety and efficacy of the combination regimen have not yet been definitively established.

MacKinnon and colleagues conducted a meta-analysis of 21 randomized, controlled trials of an ACE inhibitor plus an ARB in patients with chronic diabetic or nondiabetic (e.g. IgA nephropathy or glomerulonephritis) proteinuric renal disease $(n=654)$. The duration of treatment was generally between 1 month and 4 months.

Addition of an ARB to an ACE inhibitor ameliorated proteinuria more effectively than an ACE inhibitor alone, in both the diabetic (weighted mean difference $210 \mathrm{mg} /$ day, $95 \%$ Cl 84-336 mg/day) and nondiabetic (weighted mean difference $582 \mathrm{mg} /$ day, $95 \% \mathrm{Cl} 371-$ $793 \mathrm{mg} /$ day) subgroups. Overall, combination therapy was associated with a small increase in serum potassium levels and a slight drop in glomerular filtration rates. The analysts determined these changes to be clinically insignificant, and therefore concluded that concomitant treatment with an ACE inhibitor and an ARB safely improves proteinuria in the short term. The long-term relevance of this improvement to preservation of renal function remains to be determined.

Original article MacKinnon $\mathrm{M}$ et al. (2006) Combination therapy with an angiotensin receptor blocker and an ACE inhibitor in proteinuric renal disease: a systematic review of the efficacy and safety data. Am J Kidney Dis 48: 8-20

\section{Variation in PTH measurements with different immunoassays}

NKF/KDOQI guidelines advise that serum 'intact' parathyroid hormone (PTH) concentrations should be maintained at between $150 \mathrm{ng} / \mathrm{l}$ and $300 \mathrm{ng} / \mathrm{l}$ in patients with stage 5 chronic kidney disease (CKD). This recommended range was established using the Allegro PTH assay, and the guideline authors acknowledge that when some newer, more sensitive assays are used, target PTH values should be approximately halved (75-150 $\mathrm{ng} / \mathrm{l})$. A recent study has shown, however, that this estimated correction factor is not sufficiently specific; different immunoassays yield widely disparate PTH values, so therapeutic targets need to be tailored to individual methods.

Using 47 serum pools from dialysis patients in France, Souberbielle and co-workers compared PTH values determined using the Allegro assay with those achieved using 15 other commercially available immunoassays. The non-Allegro assays yielded PTH values ranging from almost half to over two times those provided by the Allegro assay.

The authors propose that either assayspecific PTH limits be defined when guidelines are next updated, or correcting factors be applied to measured PTH concentrations to convert them to 'Allegro PTH values'. Regular review, and adjustment if necessary, of recommended PTH thresholds should prevent assay type from influencing therapeutic manipulation of PTH levels in CKD patients.

Original article Souberbielle J-C et al. (2006) Inter-method variability in PTH measurement: implication for the care of CKD patients. Kidney Int 70: 345-350 